\title{
Potential role of upregulated microRNA-146b and -21 in renal fibrosis
}

\author{
GUANGDA XIN $^{1}$, GUANGYU ZHOU ${ }^{1}$, XIAOFEI ZHANG ${ }^{2}$ and WANNING WANG ${ }^{3}$ \\ Departments of ${ }^{1}$ Nephrology and ${ }^{2}$ Pediatrics, China-Japan Union Hospital of Jilin University, Changchun, Jilin 130033; \\ ${ }^{3}$ Department of Nephrology, The First Bethune Hospital of Jilin University, Changchun, Jilin 130021, P.R. China
}

Received July 28, 2016; Accepted May 9, 2017

DOI: $10.3892 / \mathrm{mmr} .2017 .6929$

\begin{abstract}
The aim of the present study was to identify candidate microRNAs (miRNAs) involved in the progression of renal fibrosis. Dataset GSE42716 of miRNAs extracted from kidneys from mice with unilateral ureteral obstruction (UUO), and mice without UUO was downloaded from the Gene Expression Omnibus database. The Limma package was used to identify differential expression between mice with and without UUO. Renal disease-related miRNAs were predicted based on the miRWalk database. Thereafter, candidate miRNAs were screened by taking the intersection of differentially expressed miRNAs and predicted miRNAs, followed by screening of target genes using miRWalk and transcription factors using the TransmiR database. An integrative regulatory network was constructed using Cytoscape software. Enrichment analysis of target genes was also performed. In total, 76 differentially expressed miRNAs were identified in kidneys with UUO compared with the normal samples based on dataset and 9 miRNAs were identified as related to renal disease from the miRWalk database. A Venn diagram revealed two overlapping upregulated miRNAs; miR-146b and miR-21. Transcription factor $N F K B 1$ may activate miR-146b and $A K T$ may activate miR-21. In addition, miR-21 had a regulatory effect on $I F N G$ expression and miR-146b may regulate the expression of $B C L 2$, $P T E N$ and $I F N G$. Furthermore, target genes of miR-146b and miR-21 were significantly enriched in 14 Gene Ontology terms including regulation of cell proliferation (e.g., BCL2, PTEN and $I F N G$ ). Overexpression of miR-21 may be activated by $A K T 2$ and contribute to renal fibrosis by negatively regulating IFNG expression. Furthermore, miR-146b may be activated by $N F K B 1$ and subsequently reduce the expression of $B C L 2$, $P T E N$ and $I F N G$ in the progression of renal fibrosis.
\end{abstract}

Correspondence to: Dr Guangyu Zhou, Department of Nephrology, China-Japan Union Hospital of Jilin University, 126 Xiantai Street, Changchun, Jilin 130033, P.R. China

E-mail: zhougy@jlu.edu.cn

Key words: renal fibrosis, unilateral ureteral obstruction, microRNA-21, microRNA-146b, cell proliferation

\section{Introduction}

Renal fibrosis is the result of abnormal assembly of the extracellular matrix, which ultimately leads to renal failure progression, and advanced and end-stage renal disease (1). Renal fibrosis covers four overlapping stages: The fibrogenic stage, activation, expansion and progression (2). Transforming growth factor- $\beta(T G F-\beta)$ signaling is the important mediator in the progression of renal fibrosis via inducing the extracellular matrix to form renal scarring (3). Mitogen-activated protein kinase $(M A P K)$ serves a role in the extracellular matrix in renal fibrosis as being downstream of $T G F \beta$ (4). The underlying molecular mechanisms (e.g. $T G F \beta$ and $M A P K$ signaling) are suggested as therapeutic targets of renal fibrosis (5).

MicroRNAs (miRNAs), characterized as noncoding RNAs of 18-25 nucleotides, are revealed to take part in the regulation of $T G F-\beta$-induced renal fibrosis (6). miR-21 is the $T G F \beta$-induced miRNA, which is upregulated in tubular epithelial cells and promotes renal fibrosis (7). However, miR-29 can be inhibited by $T G F-\beta$, thereby facilitating the extensive accumulation of the extracellular matrix in renal fibrosis (8). In addition, miRNAs serve fundamental roles in tissue fibrosis by either blocking the translation or inducing the degradation of target mRNAs, therefore targeting specific miRNAs may lead to novel therapeutic methods for treating renal fibrosis (9).

Unilateral ureteral obstruction (UUO) is a representative model for researching renal fibrosis resulting from non-inflammatory surgical insult (10). Previously, mice were treated with UUO to identify the involved miRNAs in renal fibrosis (11). Using a different analysis process, the current study aimed to identify candidate miRNAs specifically associated with renal fibrosis based on microarray dataset GSE42716, followed by screening of upstream transcription factors and target genes of the candidate miRNAs. Additionally, enrichment analysis of target genes was also performed in order to explain the involvement of candidate miRNAs in renal fibrosis. The workflow of the present study can be seen in Fig. 1 .

\section{Materials and methods}

Microarray data of miRNA. Microarray dataset GSE42716 of miRNA extracted from kidneys from four mice with UUO and four matched mice without UUO were downloaded from the 
Gene Expression Omnibus database (12). These microarray expression profiling data were previously investigated using GPL10834 Agilent-021828 Unrestricted Mouse miRNA Microarray (V2, Agilent Technologies, Inc., Santa Clara, CA, USA). All animal studies have been approved by Keio University Animal Ethics Committee (Shinjuku-ku, Tokyo, Japan) and performed in accordance with the ethical standards.

Data processing and screening of differentially expressed miRNAs. The raw expression data on probe-level were converted into recognizable miRNA expression data, followed by median normalization and $\log _{2}$ transformation using Affy package in $\mathrm{R}$ (13). The expression values of probes corresponding to each miRNA were averaged to obtain the expression level of the miRNA. Differentially expressed miRNAs in mice with UUO compared with the normal mice were screened with the criteria of $\log _{2}$ fold-change (FC) $\mid>1$ and false discovery rate (FDR) $<0.05$ using Limma package in R (14).

Screening of candidate miRNAs, their target genes and transcription factors. The miRNAs related to renal disease were predicted using miRWalk database (15). Then, the candidate miRNAs specifically associated with renal fibrosis were screened by taking the intersection of differentially expressed miRNAs identified in the present study and the predicted miRNAs using the miRWalk database. The persuasive target genes of candidate miRNAs were subsequently predicted using the miRWalk database, together with screening of upstream transcription factors targeting candidate miRNAs using the TransmiR database (16).

Integrative regulatory network construction. The interacting pairs among target genes were identified using the Search Tool for Retrieval of Interacting Genes database with the combined score $>0.4$ (17), followed by construction of integrative regulatory network among transcription factors, miRNAs and target genes using Cytoscape software (version 3.0.1; http://www. cytoscape.org/) (18).

Enrichment analysis of target genes from the integrative regulatory network. To reveal the potential biological functions or pathways that may be changed by the differentially expressed miRNAs, Gene Ontology (GO) term (19) and Kyoto Encyclopedia of Genes and Genomes (KEGG) enrichment analysis (20) were separately performed for the target genes of candidate miRNAs using Database for Annotation, Visualization and Integrated Discovery (DAVID) database (21) and KEGG Orthology Based Annotation System (KOBAS) tool (22) with the criterion of $\mathrm{P}<0.05$.

\section{Results}

Differentially expressed miRNAs. With the criteria of $\log _{2} \mathrm{FCl}$ $>1$ and FDR $<0.05,76$ differentially expressed miRNAs were obtained, including 15 downregulated and 61 upregulated miRNAs in kidneys with UUO compared with the normal samples.

Screened candidate miRNAs, target genes and their transcription factors. A total of nine miRNAs were predicted to

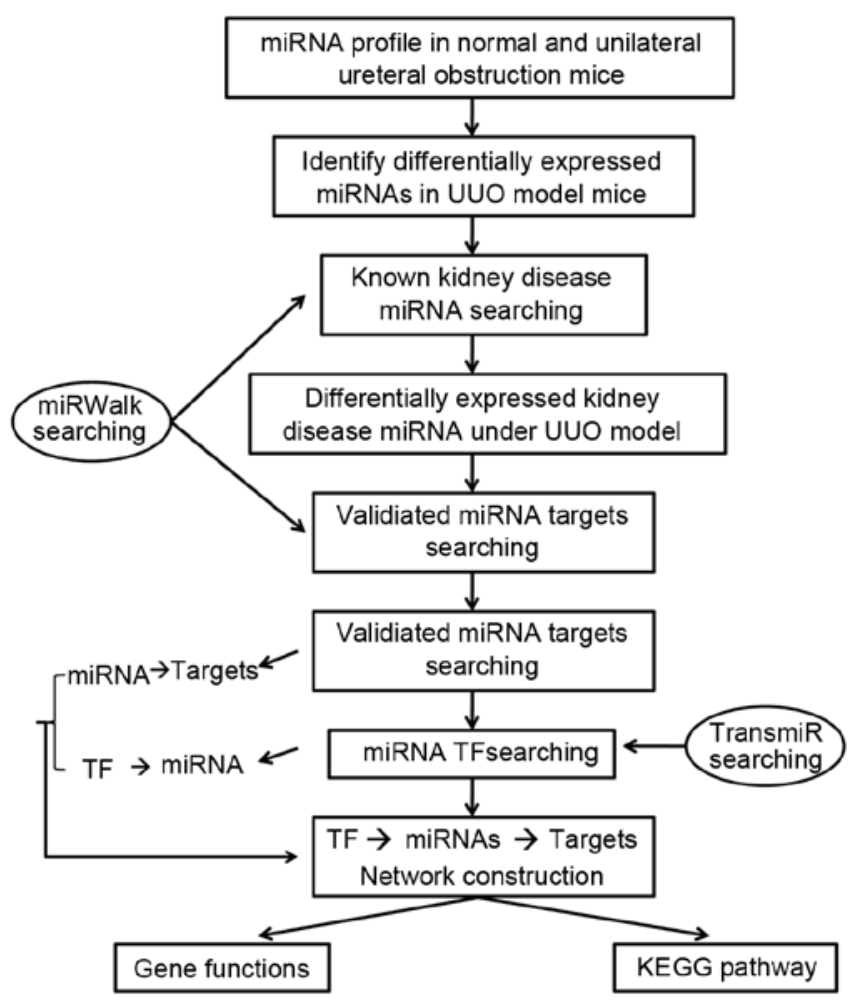

Figure 1. Workflow of the present study. miRNA, microRNA; UUO, unilateral ureteral obstruction; TF, transcription factor; KEGG, Kyoto Encyclopedia of Genes and Genomes.

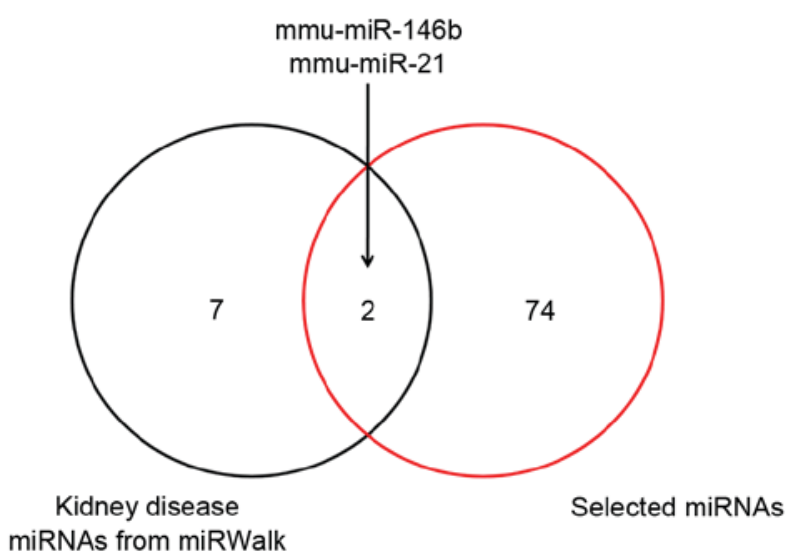

Figure 2. Venn diagram of the differentially expressed microRNAs and renal disease-related miRNAs. miRNAs, microRNAs.

be associated with renal disease using the miRWalk database. By taking the intersection of the nine predicted miRNAs and 76 differentially expressed miRNAs analyzed by a Venn diagram, upregulated miR-146b and miR-21 were identified as candidate miRNAs specifically associated with the disease of renal fibrosis (Fig. 2). Then, 74 target genes of miR-146b and 587 target genes of miR-21 were screened using the miRWalk database, followed by identification of transcription factor NFKB1 activating miR-146b and activating miR-21 using the TransmiR database (Table I).

Integrative regulatory network analysis. The interactions between target genes, regulatory effect of miRNAs on the 
Table I. Upstream TFs of miR-146b and miR-21.

\begin{tabular}{|c|c|c|c|c|c|}
\hline TF & Entrezid & $\operatorname{miR}$ & Active & Pmid & Organism \\
\hline$N F K B 1$ & 4790 & miR-146b & Activation & 21981419 & Mouse \\
\hline Gfil & 14581 & miR-21 & Repression & 19278956 & Mouse \\
\hline$A K T$ & 11651 & miR-21 & Activation & 20404348 & Mouse \\
\hline
\end{tabular}

$\mathrm{TF}$, transcription factors; miR, microRNA.

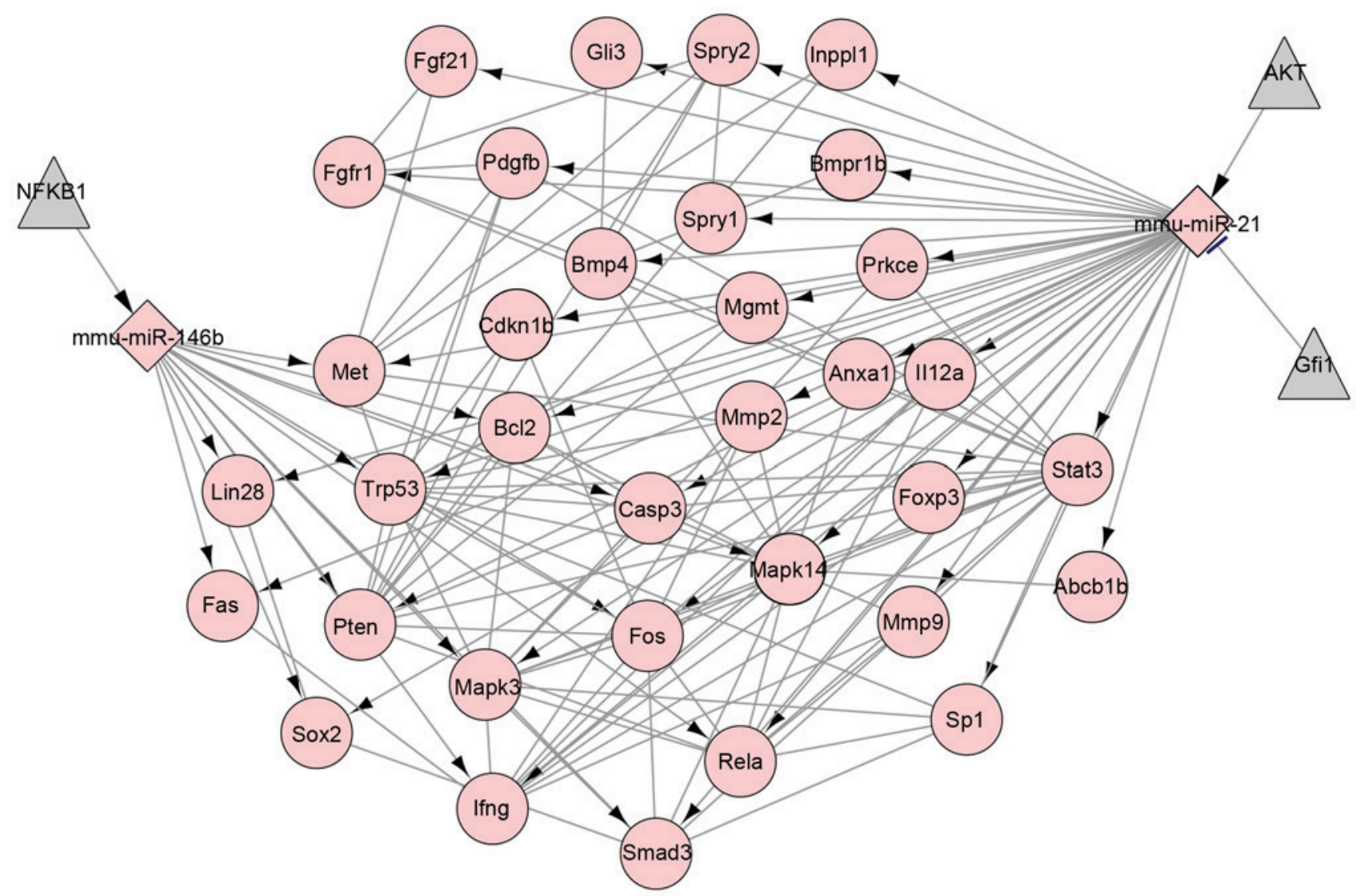

Figure 3. Integrative regulatory network among miRNAs, their target genes and transcription factors. Pink rhombuses represent miRNAs; Pink circles represent target genes; Gray triangles represent transcription factors. Between transcription factors and miRNAs, arrows represent activation and -I represents suppression. Edges between target genes represent interactions. miRNAs, microRNAs.

target genes and transcription factors on miRNAs were presented in the integrative regulatory network which included 39 nodes representing target genes, 140 edges representing interactions between genes, two miRNAs and three transcription factors (Fig. 3). Based on the regulatory network, miR-21 had a regulatory effect on $I F N G$ expression and miR-146b may negatively regulate the expression of BCL2, PTEN and IFNG.

GO functions and KEGG pathways enrichment analysis. Enrichment analysis using DAVID and KOBAS databases revealed the target genes of miR-146b and miR-21 were significantly enriched in $14 \mathrm{GO}$ terms including regulation of cell proliferation ( $\mathrm{P}=1.2 \mathrm{E}-17$, such as $B C L 2, P T E N$ and $I F N G)$, and two KEGG pathways of the TGF- $\beta$ signaling pathway $(\mathrm{P}=2.78 \mathrm{E}-03)$ and the $M A P K$ signaling pathway $(\mathrm{P}=1.11 \mathrm{E}-13$; Table II).

\section{Discussion}

miRNAs have been previously revealed to serve an important role in initiating and promoting the pathologic processes in the progression and development of renal fibrosis (23). By analyzing the microarray data of miRNAs, the current study obtained 76 differentially expressed miRNAs in mice with UUO compared with the control mice. Based on the miRWalk database, nine miRNAs were predicted to be associated with renal disease. By taking the interaction of the 76 differentially expressed miRNAs and 7 renal disease-related miRNAs, miR-146b and miR-21 were identified to be candidate miRNAs. Additionally, NFKB1 may activate miR-146b and AKT may activate miR-21. Furthermore, target genes (e.g. BCL2, PTEN and $I F N G$ ) of miR-146b were primarily involved in the regulation of cell proliferation. 
Table II. The top 5 significantly enriched GO terms and KEGG pathways of genes targeted by miR-146b and miR-21.

\begin{tabular}{|c|c|c|}
\hline Term & P-value & Enriched genes \\
\hline \multicolumn{3}{|l|}{ GO terms } \\
\hline $\begin{array}{l}\text { GO:0042127 regulation of } \\
\text { cell proliferation }\end{array}$ & $1.2 \times 10^{-17}$ & $\begin{array}{l}\text { TRP53, BMP4, FGFR1, PDGFB, INPPL1, SOX2, ANX1, SMAD3, } \\
\text { FGF21, FOXP3, PTEN, GLI3, SPRY2, SPRY1, CASP3, CDKN1B, } \\
\text { BCL2, IFNG, IL12A }\end{array}$ \\
\hline $\begin{array}{l}\text { GO:0008285 negative regulation } \\
\text { of cell proliferation }\end{array}$ & $5.7 \times 10^{-11}$ & $\begin{array}{l}\text { BMP4, TRP53, SPRY2, CASP3, SPRY1, CDKN1, INPP1, BCL2, } \\
\text { FOXP3, PTEN, GLI3 }\end{array}$ \\
\hline $\begin{array}{l}\text { GO:0010628 positive regulation } \\
\text { of gene expression }\end{array}$ & $7.5 \times 10^{-9}$ & $\begin{array}{l}\text { BMP4, TRP53, FOS, SP1, RELA, MAPK14, IFNG, SOX, SMAD3, } \\
\text { FOXP3, GLI3, STAT3 }\end{array}$ \\
\hline $\begin{array}{l}\text { GO:0007167 enzyme linked } \\
\text { receptor protein signaling pathway }\end{array}$ & $8.8 \times 10^{-9}$ & $\begin{array}{l}\text { BMP4, TRP53, FGFR1, PDGFB, MET, SMAD3, FGF21, BMPR1B, } \\
\text { PTEN, STAT3 }\end{array}$ \\
\hline $\begin{array}{l}\text { GO:0042325 regulation } \\
\text { of phosphorylation }\end{array}$ & $1.5 \times 10^{-8}$ & $\begin{array}{l}\text { BMP4, SPRY2, CASP3, SPRY1, CDKN1B, PDGFB, BCL2, IFNG, } \\
\text { MET, PRKCE }\end{array}$ \\
\hline $\begin{array}{l}\text { KEGG pathways } \\
\text { mmu04350 TGF- } \beta \\
\text { signaling pathway }\end{array}$ & $2.78 \times 10^{-3}$ & BMP4, SP1, IFNG, MAPK3, SMAD3, BMPR1B \\
\hline $\begin{array}{l}\text { mmu04010 MAPK } \\
\text { signaling pathway }\end{array}$ & $1.11 \times 10^{-13}$ & $\begin{array}{l}\text { TRP53, FOS, FGFR1, CASP3, PDGFB, RELA, MAPK14, MAPK3, } \\
\text { FGF21, FAS }\end{array}$ \\
\hline
\end{tabular}

GO, Gene Ontology; KEGG, Kyoto Encyclopedia of Genes and Genomes; miR, microRNA; TGF- $\beta$, transforming growth factor- $\beta$; MAPK, mitogen-activated protein kinase.

Highly expressed miR-21 is positively correlated with kidney fibrosis (23). The overexpression of miR-21 is induced by $T G F-\beta$ signaling (7), which regulates the deposition and contraction of extracellular matrix in renal fibrosis $(6,8,24)$. Similarly, in the present study, miR-21 was identified with significant upregulation. Besides, based on the regulatory network, miR-21 had a regulatory network on the expression of IFNG. Previously, administration of IFNG was demonstrated to reduce collagen III and IV expression and inhibit renal fibrosis in rats with subtotal nephrectomy (25). Thus, the overexpressed miR-21 may lead to inactive IFNG, thereby promoting renal fibrosis. Additionally, AKT may activate miR-21 from the regulatory network. In the mouse model of UUO, AKT2 expression dramatically increases and $A K T 2$ knockout inhibits UUO-induced activation of epithelial-to-mesenchymal transition and kidney fibrosis (26). It therefore can be inferred that the overexpressed miR-21 in mice with UUO may result from upregulated $A K T 2$ and inversely negatively regulate $I F N G$ expression, which may make a contribution to renal fibrosis.

In addition, miR-146b was also identified as upregulated in mice with UUO, which had not been reported previously. In the remodeling of atrial fibrosis, miR-146b is overexpressed and the transfection of miR-146b into cardiac fibroblasts markedly reduces expression of metallopeptidase inhibitor 4 and reversely increases collagen content (27). In the present study, miR-146b may negatively regulate the expression of BCL2, PTEN and IFNG, which were enriched in the GO terms of regulation of cell proliferation. Enhancive cell apoptosis and proliferation may contribute to the progression of renal fibrosis and their inhibition may help ameliorate renal fibrosis $(28,29)$. The expression of anti-apoptotic protein BCL2 gradually reduced with time following UUO, thereby contributing to increased cell apoptosis and renal fibrogenesis (30). In a previous study, loss of PTEN expression, accompanied by increased $T G F-\beta$ signaling, led to failed differentiation and caused fibrosis in the proximal tubules (31). Additionally, IFNG exerts an inhibitory effect on renal fibrosis in rats with subtotal nephrectomy (25). Thus, the overexpressed miR-146b in mice with UUO may contribute to renal fibrosis via suppressing the expression of BCL2, PTEN and IFNG. In addition, transcription factor $N F K B 1$ may activate miR-146b. NFKB can be upregulated by the pro-fibrotic cytokines and serve a role in the obstruction-induced cell apoptosis renal fibrosis (32). Therefore, miR-146b may be activated by NFKBI and subsequently reduce the expression of $B C L 2, P T E N$ and $I F N G$, contributing to renal fibrosis. However, further research should be conducted to validate these results.

In summary, based on the microarray data of miRNAs, the present study identified two candidate miRNAs, miR-146b and miR-21. The overexpression of miR-21 was in accordance with the previous reports $(7,23)$. However, the current study further indicated that the overexpressed miR-21 in mice with UUO may be activated by $A K T 2$ and contribute to renal fibrosis via negatively regulating $I F N G$ expression. Notably, the study also obtained another markedly expressed miRNA in mice with UUO, namely miR-146b. Furthermore, miR-146b may be activated by $N F K B 1$ and subsequently reduce the expression of BCL2, PTEN and IFNG in the progression of renal fibrosis. These present findings suggested the potential usage of miR-146b and miR-21 as diagnostic and therapeutic biomarkers for renal fibrosis. However, further studies should be designed to confirm these results. 


\section{Acknowledgements}

The present study was supported by the Natural Science Foundation of China (grant no. 81370810) and the Science and Technology Development Plan Project of Jilin Province (grant no. 20160101059JC).

\section{References}

1. Liu Y: Renal fibrosis: New insights into the pathogenesis and therapeutics. Kidney Int 69: 213-217, 2006.

2. Liu Y: Cellular and molecular mechanisms of renal fibrosis. Nat Rev Nephrol 7: 684-696, 2011.

3. Chung AC and Lan HY: Molecular mechanisms of TGF- $\beta$ signaling in renal fibrosis. Current Pathobiology Reports 1: 291-299, 2013

4. Stambe C, Atkins RC, Tesch GH, Masaki T, Schreiner GF and Nikolic-Paterson DJ: The role of p38alpha mitogen-activated protein kinase activation in renal fibrosis. J Am Soc Nephrol 15: 370-379, 2004

5. Boor P, Ostendorf T and Floege J: Renal fibrosis: Novel insights into mechanisms and therapeutic targets. Nat Rev Nephrol 6 : 643-656, 2010.

6. Chung AC, Huang XR, Meng X and Lan HY: miR-192 mediates TGF-beta/Smad3-driven renal fibrosis. J Am Soc Nephrol 21: $1317-1325,2010$

7. Zhong X, Chung AC, Chen HY, Meng XM and Lan HY: Smad3-mediated upregulation of miR-21 promotes renal fibrosis. J Am Soc Nephrol 22: 1668-1681, 2011.

8. Qin W, Chung AC, Huang XR, Meng XM, Hui DS, Yu CM Sung JJ and Lan HY: TGF- $\beta / \mathrm{Smad} 3$ signaling promotes renal fibrosis by inhibiting miR-29. J Am Soc Nephrol 22: 1462-1474, 2011.

9. Jiang X, Tsitsiou E, Herrick SE and Lindsay MA: MicroRNAs and the regulation of fibrosis. FEBS J 277: 2015-2021, 2010.

10. Ishidoya S, Morrissey J, Mccracken R, Reyes A and Klahr S: Angiotensin II receptor antagonist ameliorates renal tubulointerstitial fibrosis caused by unilateral ureteral obstruction. Kidney Int 47: 1285-1294, 1995.

11. Morizane R, Fujii S, Monkawa T, Hiratsuka K, Yamaguchi S, Homma $\mathrm{K}$ and Itoh $\mathrm{H}$ : miR-34c attenuates epithelial-mesenchymal transition and kidney fibrosis with ureteral obstruction. Sci Rep 4: 4578, 2014

12. Barrett $\mathrm{T}$ and Edgar R: Gene expression omnibus: Microarray data storage, submission, retrieval, and analysis. Methods Enzymol 411: 352-369, 2006.

13. Gautier L, Cope L, Bolstad BM and Irizarry RA: affy-analysis of Affymetrix GeneChip data at the probe level. Bioinformatics 20 . 307-315, 2004

14. Smyth GK, Limma: Linear models for microarray data, in Bioinformatics and computational biology solutions using $\mathrm{R}$ and Bioconductor., Springer. pp397-420, 2005.

15. Dweep H, Sticht C, Pandey P and Gretz N: miRWalk-database: Prediction of possible miRNA binding sites by 'walking' the genes of three genomes. J Biomed Inform 44: 839-847, 2011.

16. Wang J, Lu M, Qiu C and Cui Q: TransmiR: A transcription factor-microRNA regulation database. Nucleic Acids Res 38 (Database issue): D119-D122, 2010.
17. Franceschini A, Szklarczyk D, Frankild S, Kuhn M, Simonovic M, Roth A, Lin J, Minguez P, Bork P, von Mering C and Jensen LJ: STRING v9. 1: Protein-protein interaction networks, with increased coverage and integration. Nucleic Acids Res 41 (Database issue): D808-D815, 2013.

18. Kohl M, Wiese S and Warscheid B: Cytoscape: software for visualization and analysis of biological networks, in Data Mining in Proteomics, Springer. pp291-303, 2011.

19. Gene Ontology Consortium, Blake JA, Dolan M, Drabkin H, Hill DP, Li N, Sitnikov D, Bridges S, Burgess S, Buza T, et al: Gene Ontology annotations and resources. Nucleic Acids Res 41 (Database issue): D530-D535, 2013.

20. Kanehisa M, Goto S, Sato Y, Furumichi M and Tanabe M: KEGG for integration and interpretation of large-scale molecular data sets. Nucleic Acids Res 40 (Database issue): D109-D114, 2012.

21. Sherman BT, Huang da W, Tan Q, Guo Y, Bour S, Liu D, Stephens R, Baseler MW, Lane HC and Lempicki RA: DAVID Knowledgebase: A gene-centered database integrating heterogeneous gene annotation resources to facilitate high-throughput gene functional analysis. BMC Bioinformatics 8: 426, 2007.

22. Xie C, Mao X, Huang J, Ding Y, Wu J, Dong S, Kong L, Gao G, Li CY and Wei L: KOBAS 2.0: A web server for annotation and identification of enriched pathways and diseases. Nucleic Acids Res 39 (Web Server issue): W316-W322, 2011.

23. Zarjou A, Yang S, Abraham E, Agarwal A and Liu G: Identification of a microRNA signature in renal fibrosis: Role of miR-21. Am J Physiol Renal Physiol 301: F793-F801, 2011.

24. Lan HY: Diverse roles of TGF- $\beta /$ Smads in renal fibrosis and inflammation. Int J Biol Sci 7: 1056-1067, 2011.

25. Oldroyd SD, Thomas GL, Gabbiani G and El Nahas AM: Interferon-gamma inhibits experimental renal fibrosis. Kidney Int 56: 2116-2127, 1999.

26. Lan A, Zhang J, Xiao Z, Peng X, Qi Y and Du J: Akt2 is involved in loss of epithelial cells and renal fibrosis following unilateral ureteral obstruction. PLoS One 9: e105451, 2014.

27. Wang J, Wang Y, Han J, Li Y, Xie C, Xie L, Shi J, Zhang J, Yang B, Dong C and Xu M: Integrated analysis of microRNA and mRNA expression profiles in the left atrium of patients with non-valvular paroxysmal atrial fibrillation: Role of miR-146b-5p in atrial fibrosis. Heart Rhythm 12: 1018-1026, 2015.

28. Thomas GL, Yang B, Wagner BE, Savill J and El Nahas AM: Cellular apoptosis and proliferation in experimental renal fibrosis. Nephrol Dial Transplant 13: 2216-2226, 1998.

29. Tan X, Li Y and Liu Y: Paricalcitol attenuates renal interstitial fibrosis in obstructive nephropathy. J Am Soc Nephrol 17: 3382-3393, 2006.

30. Zhang G, Oldroyd SD, Huang LH, Yang B, Li Y, Ye R and El Nahas AM: Role of apoptosis and Bcl-2/Bax in the development of tubulointerstitial fibrosis during experimental obstructive nephropathy. Exp Nephrol 9: 71-80, 2001

31. Lan R, Geng H, Polichnowski AJ, Singha PK, Saikumar P, Mcewen DG, Griffin KA, Koesters R, Weinberg JM, Bidani AK, et al: PTEN loss defines a TGF- $\beta$-induced tubule phenotype of failed differentiation and JNK signaling during renal fibrosis. Am J Physiol Renal Physiol 302: F1210-F1223, 2012.

32. Misseri R and Meldrum KK: Mediators of fibrosis and apoptosis in obstructive uropathies. Curr Urol Rep 6: 140-145, 2005. 